\title{
NIMH Initiatives to Facilitate Collaborations Among Industry, Academia, and Government for the Discovery and Clinical Testing of Novel Models and Drugs for Psychiatric Disorders
}

\author{
Linda S Brady, ${ }^{*}$, Lois Winsky ${ }^{1}$, Wayne Goodman ${ }^{2}$, Mary Ellen Oliveri ${ }^{3}$ and Ellen Stover ${ }^{4}$ \\ ${ }^{1}$ Divison of Neuroscience and Basic Behavioral Science, National Institute of Mental Health, Bethesda, MD, USA; ${ }^{2}$ Divison of \\ Adult Translational Research and Treatment Development, National Institute of Mental Health, Bethesda, MD, USA; ${ }^{3}$ Divison \\ of Developmental Translational Research, National Institute of Mental Health, Bethesda, MD, USA; ${ }^{4}$ Divison of AIDS and \\ Health and Behavior Research, National Institute of Mental Health, Bethesda, MD, USA
}

\begin{abstract}
There is an urgent need to transform basic research discoveries into tools for treatment and prevention of mental illnesses. This article presents an overview of the National Institute of Mental Health (NIMH) programs and resources to address the challenges and opportunities in psychiatric drug development starting at the point of discovery through the early phases of translational research. We summarize NIMH and selected National Institutes of Health $(\mathrm{NIH})$ efforts to stimulate translation of basic and clinical neuroscience findings into novel targets, models, compounds, and strategies for the development of innovative therapeutics for psychiatric disorders. Examples of collaborations and partnerships among $\mathrm{NIMH} / \mathrm{NIH}$, academia, and industry are highlighted.
\end{abstract}

Neuropsychopharmacology Reviews (2009) 34, 229-243; doi: I0. I038/npp.2008. I25; published online 17 September 2008

Keywords: drug discovery; proof of concept; public-private partnership; preclinical efficacy; translational neuroscience; biomarkers

\section{INTRODUCTION}

Psychiatric disorders are the leading causes of disability worldwide, accounting for $37 \%$ of all healthy life years lost from disease (Lopez et al, 2006). Although available medications and nonpharmaceutical treatments are effective in reducing severity of specific symptoms for subsets of individuals with psychiatric disorders, a substantial proportion of individuals either do not respond at all or the extent of improvement does not translate into meaningful gains in functional outcomes. The reasons for these variables and often unsatisfactory results can be traced to the shortcomings of the available armamentarium. In some cases, serious side effects limit the use of otherwise effective medications, and in others, specific domains of function (eg cognitive deficits in schizophrenia) are poorly treated by available therapeutics (Agid et al, 2007; Pangalos et al,

${ }^{*}$ Correspondence: Dr LS Brady, Division of Neuroscience and Basic Behavioral Science, National Institute of Mental Health, 6001 Executive Boulevard, Room 7204, MSC 9645, Bethesda, MD 20892, USA, Tel: + 1 301 443-3563, E-mail: Ibrady@mail.nih.gov

Received 17 March 2008; revised 16 June 2008; accepted 13 July 2008
2007). The challenge of developing safer, more effective pharmacological treatments is even greater for mental disorders in children and adolescents. Because of heightened safety concerns, psychoactive drugs tested for use in children typically are those that previously have been used in adults (Vitiello, 2007). Although such a strategy is justifiable for safety reasons, it may miss opportunities for discovery of new medications that may have distinct value at earlier developmental stages.

The number of psychotherapeutic agents with unique mechanisms of action is actually quite small and has not grown appreciably in the past 2 decades (Hughes, 2008). The need for innovative treatments is compelling and urgent: 'In spite of new medications and psychosocial treatments, mental illnesses, such as schizophrenia and mood disorders, are as much of a public health challenge now as they were a century ago. Now is the time for research to set an ambitious goal of finding cures and preventive interventions for these disabling illnesses.' (Insel and Scolnick, 2006).

Despite tremendous advances in basic neuroscience and behavioral science that drive our understanding of the 
neural circuitry and neurobiological mechanisms underlying psychiatric disorders and significant increases in pharmaceutical research and development funding, there is a dearth of new mechanism of action therapeutics in the drug discovery pipeline (for recent reviews, see Brady et al, 2008; Carpenter and Koenig, 2008; Conn and Roth, 2008; Mathew et al, 2008). The significant challenges facing the pharmaceutical and biotechnology industry to produce new small molecule drugs for mental disorders, however, are not unique to psychiatric drug development. The high cost of developing novel drugs, the high attrition rate of candidate therapeutics during development and clinical testing, and adverse effects contribute to the high rate of failure of new compounds in clinical trials. In 2007, the number of novel drugs approved by the US Food and Drug Administration (FDA) for all diseases areas was at its lowest point since 1983; only 17 new molecular entities were approved (Hughes, 2008). Despite the unmet need for innovative treatments, only one of the new drugs, lisdexamfetamine (Vyvanse) was approved for a psychiatric indicationattention-deficit/hyperactivity disorder (ADHD).

\section{DRUG DISCOVERY, DEVELOPMENT, AND TRANSLATION}

The goal of the Institute of Medicine (IOM) Forum on Drug Discovery, Development and Translation is 'to increase the number, quality, and value of new drugs by making the drug development process faster, safer, more efficient, and more focused on the needs of patients' (http://www.iom. edu/CMS/3740/24155/39927.aspx). Clearly, there is an imperative need to quickly transform basic research discoveries into drugs, treatments, or methods for prevention to improve health. The work of the forum builds on earlier efforts of the IOM to identify two major obstacles or 'translational blocks' that impede efforts to rapidly apply science to better human health. One roadblock (designated T1) occurs in 'the transfer of new understandings of disease mechanisms gained in the laboratory into the development of new methods for diagnosis, therapy, and prevention and their first testing in humans'. Another of the translational blocks (designated T2) occurs in 'the translation of results from clinical studies into everyday clinical practice and health decision making' (Sung et al, 2003).

The 'valley of death' in drug discovery exemplifies the gap between upstream research on genes, proteins, and biological pathways and downstream proof of conceptvalidation that a target is relevant to treating a disease, that the target or mechanism can be modulated by small molecule compounds, and that the compounds have a desirable profile and are likely to be safe in humans (Moran, 2007). The National Institutes of Health (NIH) has established a number of translational research and publicprivate partnership programs to bridge the 'valley of death' by providing a vehicle for industry and academic scientists to pool intellectual and material resources to accelerate the discovery and testing of novel chemical entities.

This article presents an overview of the National Institute of Mental Health (NIMH) programs and resources to address the challenges and opportunities in psychiatric drug development starting at the point of discovery through the early phases of translational research (T1). We highlight NIMH and selected NIH efforts to stimulate translation of basic and clinical neuroscience findings into novel targets, models, compounds, and strategies for the development of innovative therapeutics for psychiatric disorders (Figure 1; Table 1). The article by Wang PS et al (2008) continues this discussion through the later stages of translational research (T2), highlighting research programs that bridge bench to practice in schizophrenia and psychotic disorders.

\section{NIMH EFFORTS IN SUPPORT OF DRUG DISCOVERY AND THE DEVELOPMENT OF PUBLIC-PRIVATE PARTNERSHIPS}

Over the past 5-10 years, NIMH Strategic Plans and National Advisory Mental Health Council workgroup reports have identified scientific priorities and opportunities to shape the Institute's portfolio in drug discovery and development. 'With greater understanding about the

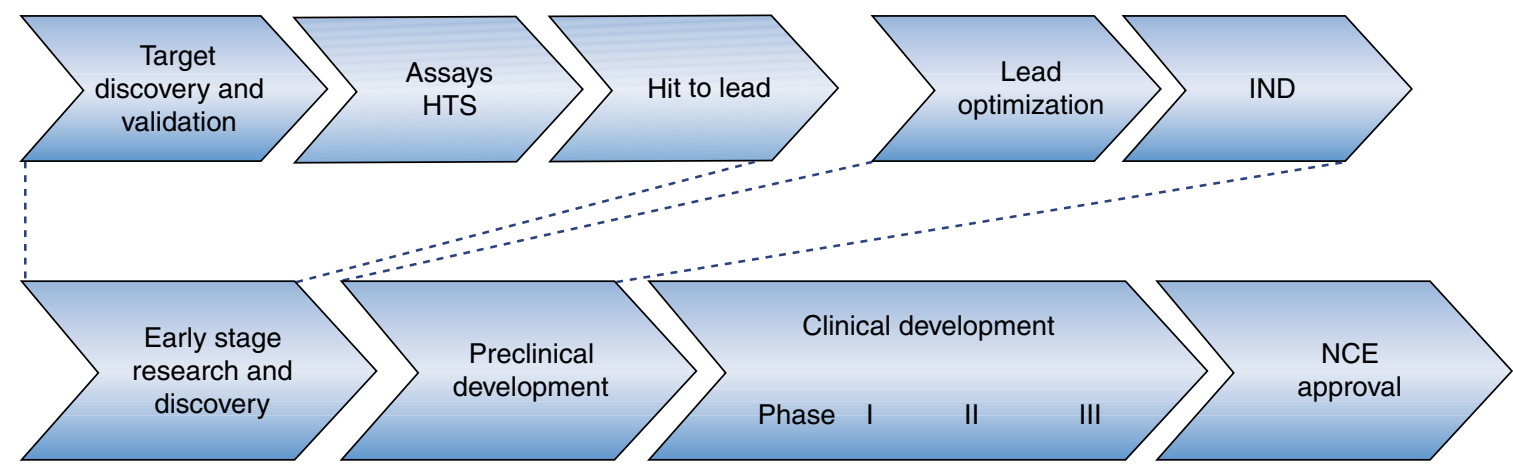

Figure 1. Drug discovery and development process. 
brain mechanisms involved in memory, decision making, and emotional responses to traumatic events, researchers are revisiting and redesigning many of the treatments currently in use for mental disorders. Translation of basic science findings into innovative behavioral and pharmacological treatments that can either correct or compensate for brain dysfunctions will yield more effective treatments' (Breaking Ground, Breaking Through: The Strategic Plan for Mood Disorders Research; Pathways to Health: Charting the Science of Brain, Mind, and Behavior, http://www.nimh. nih.gov/about/strategic-planning-reports/). In response to this charge, and in addition to its traditional role of evaluating the efficacy and effectiveness of currently available medications, the NIMH launched a broad network of programs and initiatives aimed at increasing the likelihood and speed of development of novel treatments for mental disorders. This effort resulted from the recognized public health need for a new generation of innovative therapeutics for psychiatric disorders, a first generation of medications for orphan diseases such as fragile $\mathrm{X}$ syndrome and other developmental disorders that share clinical phenotypes and deficits in core domains of cognitive function, and the new emphasis on the developmental etiology of psychiatric disorders. In addition to $\mathrm{NIMH}$ initiatives, the Institute is highly invested in larger scale NIH efforts related to both the NIH Roadmap for Biomedical Research (http://nihroadmap.nih.gov/) and the NIH Blueprint for Neuroscience Research (http:// neuroscienceblueprint.nih.gov/) in support of drug discovery and tool development.
The following sections provide an overview of the breadth of NIMH programs and initiatives, along with relevant NIH initiatives, in support of target, model, and drug discovery extending to first in human and clinical proof of concept studies (Figure 1; Table 1). A description of ongoing and recently completed large clinical trials supported by NIMH is beyond the scope of this article and can be found at http://www.nimh.nih.gov/studies/index.cfm.

\section{INNOVATIVE MODELS FOR UNDERSTANDING PATHOPHYSIOLOGY AND TESTING NOVEL THERAPEUTICS}

One of the challenges for psychiatric drug discovery is the pressing need to develop innovative models to understand the pathophysiology of psychiatric disorders and to predict the efficacy of novel treatments. Several recent articles assessed the reliability of models to predict efficacy of compounds for the treatment of schizophrenia, depression, and anxiety disorders (Carpenter and Koenig, 2008; Conn and Roth, 2008). In one study, nearly 500 compounds (140 antipsychotic, 211 antidepressant, and 143 anxiolytic) were classified based on their molecular target(s) and evaluated based on preclinical validation, whether preclinical studies predicted clinical efficacy, and whether the compound displayed greater efficacy than 'conventional treatment' (Conn and Roth, 2008). The predictive value of existing preclinical models was variable, with modest to poor ability to predict the efficacy of novel mechanism of action

\begin{tabular}{|c|c|}
\hline \multirow{2}{*}{$\begin{array}{l}\text { Drug discovery and development Pipeline } \\
\text { Early stage research and discovery }\end{array}$} & \multirow[t]{2}{*}{ NIMH or NIH programs or initiatives ${ }^{a}$} \\
\hline & \\
\hline $\begin{array}{l}\text { Target discovery and validation: } \\
\text { genes, drug targets, preclinical models, } \\
\text { research tools }\end{array}$ & $\begin{array}{l}\text { Investigator initiated research } \\
\text { Selected Resources: } \\
\text { - Chemical Synthesis and Drug Supply Program } \\
\text { - GAIN, GEI } \\
\text { - KOMP } \\
\text { - Mouse Models (PAR-08-158) } \\
\text { - PET Imaging (PA-06-463) } \\
\text { - Psychoactive Drug Screening Program } \\
\text { - SBIR(PA-08-142,PA-08-137,PA-06-016,PA-06-375) }\end{array}$ \\
\hline - Assays & $\begin{array}{l}\text { Assay development (PAR-08-024) } \\
\text { HTS Assay (PAR-08-035) } \\
\text { Fast Track Entry Process (NOT-RM-07-012) } \\
\text { SBIR (PA-08-142,PA-08-001) }\end{array}$ \\
\hline - HTS & $\begin{array}{l}\text { MLSCN/MLPCN } \\
\text { NIH Clinical Collection } \\
\text { SBIR/STTR (PA-08-001,PA-08-002) }\end{array}$ \\
\hline - Hit to lead & \\
\hline $\begin{array}{l}\text { Hit generation, chemical optimization, } \\
\text { computational chemistry, SAR, } \\
\text { in vitro functional assays, data mining }\end{array}$ & $\begin{array}{l}\text { Drug Discovery for Nervous System Disorders } \\
\text { (PAR-07-048;PAR-07-049) } \\
\text { MLSCN/MLPCN } \\
\text { NCDDG/NCDDDG (PAR-08-238) } \\
\text { PubChem } \\
\text { SBIR (PA-08-142,PA-07-424) }\end{array}$ \\
\hline o Selectivity testing & $\begin{array}{l}\text { Psychoactive Drug Screening Program } \\
\text { SBIR (PA-08-142) }\end{array}$ \\
\hline
\end{tabular}


TABLE 1 Continued

\begin{tabular}{|c|c|}
\hline \multirow{2}{*}{\multicolumn{2}{|c|}{$\begin{array}{l}\text { Preclinical development } \\
\text { - Continuation of lead optimization to IND } \\
\text { filing }\end{array}$}} \\
\hline & \\
\hline $\begin{array}{l}\text { Medicinal chemistry, SAR, biology, } \\
\text { preclinical testing, proof of mechanism, } \\
\text { back up compounds }\end{array}$ & $\begin{array}{l}\text { Chemical Synthesis and Drug Supply Program } \\
\text { MLSCN/MLPCN } \\
\text { NCDDG/NCDDDG (PAR-08-238) } \\
\text { SBIR (PA-08-142) }\end{array}$ \\
\hline o Scale up synthesis and bulk supply & $\begin{array}{l}\text { NIH-RAID } \\
\text { SBIR (PA-08-142) }\end{array}$ \\
\hline o GMP synthesis & $\begin{array}{l}\text { Chemical Synthesis and Drug Supply Program } \\
\text { NIH-RAID } \\
\text { SBIR (PA-08-142) }\end{array}$ \\
\hline o Pharmacokinetics, bioanalytical assays & $\begin{array}{l}\text { NIH-RAID } \\
\text { SBIR (PA-08-142) }\end{array}$ \\
\hline $\begin{array}{ll}\text { O GLP toxicology studies supporting } \\
\text { O } & \text { IND Safety pharmacology studies }\end{array}$ & $\begin{array}{l}\text { NCDDG/NCDDDG (PAR-08-238) } \\
\text { NIH-RAID } \\
\text { SBIR (PA-08-142) } \\
\text { Toxicological Evaluation of Novel Ligands Program }\end{array}$ \\
\hline o Formulation & $\begin{array}{l}\text { NIH-RAID } \\
\text { SBIR (PA-08-142) }\end{array}$ \\
\hline \multicolumn{2}{|l|}{ Clinical development } \\
\hline $\begin{array}{l}\text { Phase I- safety: dose selection, } \\
\text { pharmacokinetics, exploratory biomarkers }\end{array}$ & $\begin{array}{l}\text { Autism Consortium/STAART } \\
\text { Biomarkers Consortium } \\
\text { CNTRICS } \\
\text { NCDDG/NCDDDG (PAR-08-238) } \\
\text { NIH Toolbox } \\
\text { SBIR } \\
\text { TURNS }\end{array}$ \\
\hline $\begin{array}{l}\text { Phase I/II - efficacy: proof of concept, } \\
\text { exploratory biomarkers }\end{array}$ & $\begin{array}{l}\text { Autism Consortium/STAART } \\
\text { Biomarkers Consortium } \\
\text { CDDG/NCDDDG (PAR-07-159) } \\
\text { CNTRICS } \\
\text { MATRICS Consensus Cognitive Battery } \\
\text { MATRICS CT } \\
\text { PROMIS } \\
\text { SBIR (PA-08-142,PA-08-137,PA-06-016, } \\
\text { PA-06-079) } \\
\text { TURNS }\end{array}$ \\
\hline
\end{tabular}

Abbreviations. CDDG, Cooperative Drug Development Group; CNTRICS, Cognitive Neuroscience Approaches to Treatment Development for Impaired Cognition in Schizophrenia; GAIN, Genetic Association Information Network; GEI, Genes, Environment and Health Initiative; KOMP, Knock Out Mouse Project; HTS, High throughput screen; MATRICS, Measurement and Treatment Research to Improve Cognition in Schizophrenia; MLSCN/MLPCN, Molecular Libraries Screening Centers Network/Molecular Libraries Probe Production Centers Network; NCDDG, National Cooperative Drug Discovery Group; NCDDDG, National Cooperative Drug Discovery and Development Group; PROMIS, Patient-Reported Outcomes Measurement Information System; SBIR, Small Business Innovation Research; NIH-RAID, $\mathrm{NIH}$-Rapid Access to Interventional Development; STAART, Studies to Advance Autism Research and Treatment; TURNS, Treatment Units for Research on Neurocognition and Schizophrenia aSee text for link

compounds in schizophrenia or depression, and slightly more reliability in predicting efficacy for anxiety disorders, but not for specific types of anxiety. In addition, a recent editorial lends weight to the argument that traditional mechanistic models of psychiatric disorders may be misleading, and calls for the use of model organisms to identify the function of genetic alleles and cellular changes that confer risk and resiliency to mental disorders (Insel, 2007).

The need for novel models that address pathology of mental disorders and discover targets for therapeutic intervention is congruent with efforts to identify genetic risk alleles and epigenetic mechanisms that code susceptibility conferred by adverse life experiences. To determine potential relationships between genes associated with brain disorders and pathology, NIMH recently issued an initiative, Mouse Models Containing Human Alleles: Novel Tools to Study Brain Function (http://grants.nih.gov/grants/ guide/pa-files/PAR-08-158.html). This initiative supports the development and characterization of novel mouse models that express gene variants and gene-dosage abnormalities that have been associated with mental disorders through whole genome association studies (WGASs) and by other means. This effort is complementary to ongoing NIH efforts aimed at providing a large array of genomic and genetic resources to the broad research community. Thus, NIH has established centralized resources of information on model organisms available to researchers such as the Knockout Mouse Project (KOMP, http://www.nih.gov/science/models/mouse/knockout/) and a formal model organism sharing policy that encourages 
the wide dissemination of genetic tools (http://www.nih.gov/science/models/). In addition, the NIH Blueprint for Neuroscience Research supports the design, creation, and characterization of Cre-recombinase expressing ('Cre-Driver') mouse lines that can be used to selectively express genes within distinct brain cells and with temporal control for studies of gene function in adult and developing brain. It is expected that these and other efforts to create and disseminate novel genetic tools (http://neuroscienceblueprint. nih.gov/neuroscience_resources/animal_models.htm) throughout the neuroscience community will be essential in linking genetic findings in mental disorders with understanding of the potential functional consequences of gene variation for brain function.

\section{RESOURCE SUPPORT FOR DRUG DISCOVERY}

The NIMH complements support of investigator-initiated grants in the area of treatment research through specific initiatives developed to stimulate and facilitate drug discovery and development initiatives. These initiatives are intended to encourage investigators interested in developing new therapeutics or novel ways to test candidate compounds, including new assays or model systems to evaluate potential efficacy and utility in the treatment of mental disorders. The initiatives span the breadth of the drug development process, from target identification and ligand discovery, to preclinical development.

The NIMH has identified several opportunities for linking molecular neuroscience with efforts to facilitate both drug and tool discovery relevant to mental disorders. To address the needs of a diverse set of researchers interested in advancing the study of novel compounds toward these goals, the NIMH established contract services for drug screening, synthesis, and preclinical toxicology studies.

The NIMH Psychoactive Drug Screening Program is a resource program that provides screening of novel psychoactive compounds for pharmacological and functional activity at cloned human or rodent CNS receptors, channels, and transporters (http://pdsp.med.unc.edu/). The contract also provides assays for predicting bioavailability and cardiovascular toxicity and supports a $\mathrm{Ki}$ database of affinity constants for ligand binding (http://pdsp.med.unc. edu/pdsp.php).

The NIMH Chemical Synthesis and Drug Supply Program synthesizes and distributes novel research chemicals, psychoactive drugs, positron emission tomography (PET) and single photon emission computed tomography (SPECT) imaging ligand precursors, and compounds that are unavailable to the scientific community from commercial sources to stimulate basic and clinical research relevant to mental health (http://nimh-repository.rti.org/). The repository contains more than 350 compounds that are available for research use, and has just begun to distribute screening plates of the inventory in 96-well format. In addition, the program has the capability to provide limited
Good Manufacturing Practice (GMP) synthesis of promising candidate compounds for use in clinical studies.

The NIMH Toxicological Evaluation of Novel Ligands Program advances the discovery of neuroimaging biomarkers by providing toxicology and safety assessment services for promising, target-selective ligands for PET, SPECT, or magnetic resonance imaging studies (MRI) in humans (http://www.sri.com/biosciences/nimh/nimh-tox.html). The program also provides limited assessment of novel psychoactive agents for clinical research and as potential therapeutics. Toxicology and safety data generated by the program are used to support exploratory Investigational New Drug (IND) applications to the FDA for human studies. The program has conducted toxicological evaluation of more than a dozen novel imaging ligands for G-proteincoupled receptors (GPCRs), transporters, and metabotropic glutamate receptor subtypes as well as pharmacokinetic and bioavailability assessment of three novel clinical candidates.

The NIH Roadmap Molecular Libraries Program has established a translational high-throughput screening (HTS) screening resource in the academic environment to improve the understanding of biology and disease mechanisms (http://nihroadmap.nih.gov/molecularlibraries/). At the core of this initiative is the Molecular Libraries Screening Centers Network (MLSCN, http://www.mli. nih.gov; the Molecular Libraries Probe Production Centers or MLPCN beginning in September 2008 as the program moves into the production phase). The MLSCN optimizes and performs HTS to identify compounds that are active in biochemical, cell-based, or model organism assays using multiple screening formats, including target-based, reporter, and phenotypic screens. Active compounds identified through initial screening of the library (the Small Molecule Repository) are optimized through a combination of structure-activity analysis and synthetic chemistry to obtain useful chemical probes that can be used to study the functions of genes, cells, and biochemical pathways. $\mathrm{NIH}$ anticipates that these projects will also facilitate the development of new drugs by providing early-stage chemical compounds that will enable researchers in the public and private sectors to validate new drug targets, which could then move into the drug development pipeline. For example, the availability of small molecule probes to characterize the biology of genes of interest, cellular processes, and proteins associated with disease processes will further advance our knowledge of nervous system pathophysiology and unveil additional targets for new therapeutic approaches to human diseases, including psychosis, depression, anxiety, pain, and aging-associated neurodegenerative disorders.

In its pilot phase, the MLSCN consortium included one intramural and nine extramural centers with expertise in assay development, HTS, chemistry, and informatics. Compounds screened by the network of centers are maintained within the Small Molecule Repository that was established in 2004 and currently has a set of approximately 
300000 compounds of specified purity, quantity, and solubility (http://mlsmr.glpg.com/MLSMR_HomePage/). The library contains structures not typically found in commercial collections or those housed in pharmaceutical companies. Sources of these unique structures include: natural products and novel compounds libraries prepared by academic investigators through the Pilot Scale Libraries program (http://mli.nih.gov/funded-research/chemicaldiversity/); compounds generated by the Centers for Chemical Methodologies and Library Development (http:// www.nigms.nih.gov/Initiatives/CMLD); and those obtained through an $\mathrm{NIH}$ solicitation (http:/grants.nih.gov/grants/ guide/notice-files/NOT-RM-07-005.html). The library of compounds has been assayed in more than 200 biological targets to date.

Data generated by the MLSCN centers including assay descriptions, chemical structures, and biological assay and profiling data for individual compounds are available in PubChem, a publicly accessible database maintained by the National Library of Medicine (http://pubchem.ncbi.nlm. nih.gov/). Investigators in the public and private sector have access to resources of the screening centers, on a competitive basis, through HTS and Assay Development initiatives (http://mli.nih.gov/), by submitting compounds to the Small Molecule Repository for screening, and through PubChem. In addition, the NIH Clinical Collection, a plated array of 450 drugs that have been in clinical testing, is available to researchers at cost (http://www.nihclinicalcollection. $\mathrm{com} /$ ) to enable benchtop screening. These compounds may serve as starting points for medicinal chemistry optimization efforts and, in some cases, may even be appropriate for use in new disease areas (ie, drug repurposing; Chong and Sullivan, 2007). Chemical probes are emerging from the MLSCN at an increasing rate for a variety of targets and cell phenotypes, many in the CNS area (Huang et al, 2007; Lewis et al, 2008; Niswender et al, 2008; Skoumbourdis et al, 2008; Titus et al, 2008). More information on this Molecular Libraries effort is available (Austin et al, 2004; Huryn and Cosford, 2008; Lazo et al, 2007).

NIH Rapid Access to Interventional Development (NIHRAID) is an NIH Roadmap pilot program intended to reduce some of the common barriers (the 'valley of death') in the small molecule development pipeline from preclinical discovery to clinical testing (http://nihroadmap.nih.gov/ $\mathrm{raid} /$ ). The goal of the NIH-RAID pilot is to make available, on a competitive basis, certain critical resources needed for the development of therapeutic agents. NIH contract services supported by the program include: scale-up production, bulk supply, and GMP manufacturing of small molecule drugs for phase I studies, formulation development, development of bioanalytical assays for pharmacokinetic studies, range finding and IND-directed toxicology, and product development planning to support IND filing. Academic investigators are invited to submit resource access applications in response to the program announcement (http://grants.nih.gov/grants/guide/pa-files/PAR-07358.html). The program does not support in vitro or in vivo testing or production of materials for studies beyond phase I. Contract services have assisted in the development of several, novel CNS therapeutic agents through the NIHRAID program with co-funding from NIMH and the neuroscience institutes. Examples of approved projects include: vasopressin 1A antagonist SRX246 for treating anxiety and depression; M1 muscarinic agonist CDD-0102A for the treatment of Alzheimer's disease; and $\kappa$-opioid receptor antagonist JDTic for treating drug abuse (cocaine relapse), depression, and schizophrenia (http://nihroadmap. nih.gov/raid/ApprovedProjects.aspx).

To maximize the potential for translating basic molecular science into treatment and tool discoveries, the Drug Discovery for Nervous System Disorders initiative specifically encourages the submission of applications aimed at drug discovery and early preclinical testing of compounds with therapeutic potential (http://grants.nih.gov/grants/guide/ pa-files/PAR-07-048.html). The initiative encourages studies aimed at design, synthesis, and preclinical testing of compounds, development of novel delivery systems, and cell-based assays for screening of candidate compounds for efficacy and/or toxicity. The announcement also encourages the development of novel assays using model organisms or behavioral systems for preliminary screening or further evaluation of candidate compounds, including in vivo models that emulate critical features of specific CNS disorders. Model development under these funding opportunities should be directed toward assessing potential efficacy rather than elucidating disease mechanisms. Applications submitted in response to this announcement are directed to Neural Drug Discovery, a Special Emphasis Review Panel convened by the Center for Scientific Review (http://www.csr.nih.gov/Roster_proto/allother_sep.asp). Projects funded through this initiative by NIMH have examined a range of promising targets for mental disorders, including subtype selective benzodiazepine ligands, peptide receptor ligands, metabotropic glutamate receptor potentiators, and GSK- $3 \beta$ inhibitors.

The discovery and application of novel imaging ligands hold promise for both facilitating drug discovery and for identifying molecular and cellular pathways implicated in psychiatric disorders as targets for treatment development. NIMH supports the development of imaging ligands through initiatives such as the Development and Application of PET and SPECT Imaging Ligands as Biomarkers for Drug Discovery and for Pathophysiological Studies of CNS Disorders. This initiative encourages applications aimed at developing novel radioligands for PET or SPECT imaging in human brain to facilitate the broad application of neuroimaging probes in pathophysiological studies, drug discovery/ development research, and in biomarker development/ qualification studies as quantifiable indicators of disease progression and treatment efficacy (http://grants.nih.gov/ grants/guide/pa-files/PA-06-461.html). Projects supported by NIMH in response to this initiative include the development of radiotracers for targets including GPCRs, transporters, glutamate receptor subtypes, and $\beta$-amyloid. 
Some of these projects have involved collaborations with private sector partners.

\section{SUPPORTING COLLABORATIONS AMONG GOVERNMENT, INDUSTRY, AND ACADEMIA FOR PRECLINICAL, FIRST IN HUMAN, AND CLINICAL STUDIES}

Small businesses have an increasingly important role in drug discovery and development. The NIMH supports small business involvement in drug discovery through the Small Business Innovation Research (SBIR) program sponsorship of several targeted initiatives aimed at the development of novel pharmacological treatments for mental disorders (http://grants.nih.gov/grants/guide/pa-files/PA-08-142.html), including novel molecular/cellular and in vivo screening assays, the commercial development of novel radioligands for PET and SPECT imaging in human brain (http:// grants.nih.gov/grants/guide/pa-files/PA-08-137.html). The program also supports initiatives to develop novel tools for investigating brain-derived GPCRs (http://grants. nih.gov/grants/guide/pa-files/PA-06-375.html), and highthroughput tools for brain research at any level of analysis, from molecules through behavior (http://grants.nih.gov/ grants/guide/pa-files/PA-08-001.html). As an example of the impact of this program, NIMH SBIR funding supported the development of SmartCube technology at PsychoGenics Inc. (Hawthorne, NY). The SmartCube technology platform employs an automated, high-throughput system for measuring physiology and behavior in live animals to rapidly evaluate novel compounds (CRISP abstract, http://crisp.cit.nih.gov/crisp/ CRISP_LIB.getdoc?textkey=7326301\&p_grant_num $=1$ R43 MH075460-01A2\&p_query $=($ psychogenics $)$ \&ticket $=57512753$ \&p_audit_session_id=293002869\&p_audit_score=19\&p_audit_ numfound=1\&p_keywords=psychogenics). PsychoGenics has now partnered with Eli Lilly and Company, Sepracor Inc., Cephalon Inc., and Sosei Company Ltd to use SmartCube in screening proprietary compounds for psychiatric disorders (http://www.psychogenics.com/pressreleases.shtml). Additional NIMH SBIR initiatives can be found at http://www.nimh.nih.gov/research-funding/smallbusiness/small-business-program-announcements-issued-bynimh.shtml.

The NIMH participates in several large grant and cooperative agreement programs aimed at fostering partnerships among NIMH, academia, and industry to advance the development and testing of fundamentally new, rationally designed medications and treatments for mental disorders. These initiatives provide a vehicle for industry and academic scientists to pool intellectual and material resources for the translation of basic science findings into the conceptualization, discovery, and evaluation of new chemical entities in preclinical, first in human, and proof of concept studies in the treatment of mental disorders. Below are descriptions of translational programs that have been an effective means for NIMH to assist the academic and private sector efforts to fill the drug discovery pipeline with novel mechanism of action compounds for the treatment of mental disorders.

The National Cooperative Drug Discovery Group (NCDDG) program was implemented in 2004 in response to the NIMH strategic plan for mood disorders to support the discovery of pharmacological agents targeting novel molecular targets implicated in the pathophysiology of mood and anxiety disorders. The goal of the NCDDG program at that time was to accelerate innovative drug discovery, the development of pharmacological tools for basic and clinical research on mental disorders or nicotine addiction, and the development and validation of models for evaluating novel therapeutics for mental disorders through encouraging scientific collaborations between academia and the private sector. Academic and/or pharmaceutical industry components of each NCDDG were charged with contributing unique scientific expertise toward the common goal of translating basic science findings into innovative pharmacological treatments. Several innovative projects involving academic and private sector partnership were supported. One project that involves collaboration between investigators at the University of Pennsylvania and Wyeth Discovery Neuroscience, Depression and Anxiety Research Group is focusing on central growth factors and cell proliferation as a novel mechanism for developing treatments for depression and anxiety (Malberg et al, 2007; CRISP abstract, http://crisp.cit.nih.gov/crisp/CRISP_LIB. getdoc?textkey $=7234053 \&$ p_grant_num $=5$ U01MH072832-03 \&p_query=\&ticket $=66493697 \& p \_$audit_session_id $=330527981$ \&p_keywords=).

Another project that involves collaboration between investigators at Emory University School of Medicine, the NIMH Intramural Research Program, the Mount Sinai School of Medicine, and GlaxoSmithKline Center for Excellence in Drug Discovery in Psychiatry has preclinical and clinical components to assess the efficacy of novel compounds. The project has focused on assessing the efficacy of corticotrophin releasing hormone receptor 1 antagonists and a substance P or neurokinin1 (NK1) receptor antagonist in preclinical models of anxiety and depression, startle models in rodents and a cytokine-induced depression model in primates (Felger et al, 2007). For the clinical component, proof of concept studies are in completion for evaluating the efficacy of the NK1 antagonist in post-traumatic stress disorder (PTSD, see NCT00211861, http://clinicaltrials.gov/ ct2/show/NCT00211861?term=NK1\&rank=1; NCT00383786, http://clinicaltrials.gov/ct2/show/NCT00383786?term $=$ NK1 \&rank=2).

In the current announcement, the NCDDG program has expanded its focus to include all mental disorders, drug, or alcohol addiction with the goal of accelerating the development of research tools for new molecular targets implicated in mental disorders, or drug or alcohol addiction, and of effective compounds for the prevention and treatment of psychiatric and addictive disorders, as well as core features of these illnesses, especially in areas of unmet medical need (http://grants.nih.gov/grants/guide/pa-files/PAR-07-159.html). 
Each partnership or group must consist of a multidisciplinary team of scientists with appropriate expertise to address the development and evaluation of novel ligands, and the development of testing models where required. NIMHrelevant NCDDG research projects can focus on one or more molecular targets and include two or more of the following areas: (1) ligand discovery for therapeutics development and as research tools (eg, imaging probes) for novel molecular targets implicated in mental illnesses; (2) preclinical testing of novel compounds in disease-based models; (3) development and validation of novel, disease-based genetic models combined with environmental or behavioral manipulations for evaluating therapeutic compounds; (4) initial GLP toxicology, safety pharmacology, and pharmacokinetics to support IND application to the FDA to begin human clinical testing, and/or (5) limited phase I studies. The program also supports the goal of developing and evaluating new cellular, circuit, genetic, or pathophysiology-based models for validation of novel targets for mental disorders. These collaborative projects have potential to facilitate novel hypotheses and approaches toward the development of more effective therapeutics with fewer adverse effects. For example, a recently funded project involves collaboration between investigators at University of North Carolina Chapel Hill, Duke University, and Wyeth Neuroscience Discovery, Schizophrenia Research Group. The project focuses on functional selectivity of compounds as a platform for drug discovery in schizophrenia (Mailman, 2007; Urban et al, 2007). On the basis of the initial selectivity profile, compounds will be evaluated by innovative technology such as 'designer receptors' (Armburster et al, 2007) and molecular profiling, and then in mouse models and a battery of behavioral tests. The goal of this strategy is to identify and prioritize compounds with novel mechanisms for further testing as potential therapeutics for schizophrenia (CRISP abstract, http://crisp.cit.nih.gov/crisp/CRISP_LIB.getdoc?textkey= 7502014\&p_grant_num $=5 \mathrm{U} 19 \mathrm{MH} 082441-02 \&$ p_query $=\&$ ticket $=$ 66493590\&p_audit_session_id=330527981\&p_keywords=).

\section{CLINICALLY ORIENTED COLLABORATIVE INITIATIVES TO TRANSITION CLINICAL CANDIDATES FROM DISCOVERY TO CLINICAL DEVELOPMENT}

NIMH has developed a number of innovative programs to foster collaborations among government, academia, and industry. One area of focus has involved the identification of key functional deficits in mental disorders and the use of standardized assessment instruments to evaluate the efficacy of novel pharmacological treatments. Selected examples of these translational initiatives are highlighted to illustrate the type and scope of work supported.

The Cooperative Drug Development Group (CDDG) program was developed in 2005 to support proof of concept studies of IND-ready compounds for the treatment of core symptoms associated with mental disorders. As with the
NCDDG, grants funded under the CDDG program foster long-term partnerships among NIMH, academia, and industry to advance the development and testing of new medications and treatments for serious mental disorder (http://grants1.nih.gov/grants/guide/pa-files/PAR05-010.html). However, in contrast to the NCDDG that is aimed at more preclinical discovery, the principal aim of the CDDG program has been the testing of novel mechanism therapeutics or approved agents in clinical populations as a mandatory element. The projects funded under the CDDG program help fill the gap between preclinical drug discovery efforts and the clinical effectiveness trial networks also supported by the NIMH. In 2008, the NCDDG and CDDG initiatives will be merged into a single program announcement, the National Cooperative Drug Discovery and Development Groups (NCDDDG) program (http://grants1. nih.gov/grants/guide/pa-files/PAR-08-238.html). A few examples of CDDG projects are described as follows.

Researchers at the Nathan Kline Institute are testing D-serine as an effective treatment for persistent negative symptoms and cognitive dysfunction in schizophrenia and in prodromal schizophrenia patients (CRISP abstract, http:// crisp.cit.nih.gov/crisp/CRISP_LIB.getdoc?textkey $=7280390$ \&p_grant_num =5U01MH074356-03\&p_query $=\&$ ticket $=$ 57659771\&p_audit_session_id=293246935\&p_keywords=). A CDDG being conducted in China is assessing an $\alpha-7$ neuronal nicotinic acetylcholine receptor agonist tropisetron as a target for pharmacotherapy in schizophrenia to improve cognitive and perceptual disturbances and symptoms in schizophrenia and to reduce nicotine use when administered in conjunction with the atypical neurolepetic, risperidone (CRISP abstract, http://crisp.cit.nih.gov/crisp/ CRISP_LIB.getdoc?textkey $=72803908$ p_grant_num $=5$ U01 MH074356-03\&p_query=\&ticket $=57659771 \&$ p_audit_session_ id=293246935\&p_keywords=).

A second initiative, the Centers for Intervention Development and Applied Research (CIDAR) program was developed to support interdisciplinary teams of basic, applied, and clinical investigators to target challenging problems in the diagnosis or treatment of mental illness. The program focused on research to (1) define predictors and understand the mechanism of treatment response in major mental disorders, (2) create and refine biomarkers to assess the presence and/or extent of mental illness, and (3) hasten the development of novel treatments for mental disorders (http://grants.nih.gov/grants/guide/notice-files/ NOT-MH-06-123.html). The goal of CIDAR is to accelerate the translation of basic and clinical research into innovations in clinical assessment and therapeutics. The following examples are CIDARs that involve adult populations.

Researchers at Emory University are identifying factors that may predict treatment response by comparing the effectiveness of three antidepressant treatments: escitalopram, a selective serotonin reuptake inhibitor; duloxetine, a serotonin norepinephrine reuptake inhibitor; and cognitive behavioral therapy in people with major depressive disorder 
(CRISP abstract, http://crisp.cit.nih.gov/crisp/CRISP_LIB. getdoc?textkey=7261375\&p_grant_num=5P50MH077083-02 \&p_query $=\&$ ticket $=57660183 \&$ \&_audit_session_id=293246935 \&p_keywords=). Investigators at the University of Cincinnati are identifying specific markers and predictors of treatment response early in the course of bipolar disorder. The organizing theme is that bipolar disorder results from dysfunctional metabolism within specific brain systems (ie the anterior limbic network) that can be monitored in response to treatments (lithium or olanzapine) using functional MRI (fMRI) and magnetic resonance spectroscopy (CRISP abstract, http://crisp.cit.nih.gov/crisp/CRISP_ LIB.getdoc?textkey=7251151\&p_grant_num=1P50MH07713801A1\&p_query $=\&$ ticket $=57661579 \& p \_$audit_session_id= 293246935\&p_keywords=). A third CIDAR at Harvard examines the progressive decline in brain neurocognitive and executive functions from prodromal to chronic schizophrenia patients. Prodromal, first-episode, and chronic schizophrenia patients will be compared to controls. Neurophysiological tests will assess changes in cognitive function across the course of the illness and results will be related to physiological and structural brain measures (fMRI, MRI, electrophysiology) and genetic markers (CRISP abstract, http://crisp.cit.nih.gov/ crisp/CRISP_LIB.getdoc?textkey $=7251650 \&$ \&_grant_num $=$ 1P50MH080272-01\&p_query=\&ticket $=57662052 \&$ p_audit_ session_id=293251683\&p_keywords=).

A related NIMH centers program, Translational Research Centers in Behavioral Science (http:/grants.nih.gov/grants/ guide/pa-files/par-04-151.html) supports the Center for Neurocognition and Emotion in Schizophrenia. This center involves research teams that integrate basic behavioral researchers and clinical researchers to facilitate meaningful and novel collaborations to translate advances in basic cognitive and affective science into clinical research studies (CRISP abstract, http://crisp.cit.nih.gov/crisp/CRISP_LIB. getdoc?textkey=7289703\&p_grant_num=5P50MH066286-05 \&p_query $=\&$ ticket $=58946466 \&$ p_audit_session_id $=297398752$ \&p_keywords=).

The NIH-supported Studies to Advance Autism Research and Treatment (STAART) network consists of eight centers across the United States. that conduct cross-disciplinary research on the scientific understanding and optimal treatment of autism (STAART, http://www.nimh.nih.gov/ research-funding/scientific-meetings/recurring-meetings/ iacc/nih-initiatives/staart/index.shtml). Two collaborations across STAART centers focus on pharmacological interventions for children and adolescents with autism. One study assesses the safety and feasibility of treating 30- to 58-month-old autistic children with fluoxetine to enhance developmental processes in core areas impacted by autism (NCT00183339, http://www.clinicaltrials.gov/ct2/show/ NCT00183339?term=NCT00183339\&rank=1). The second aims to determine the efficacy and safety of citalopram compared to placebo in the treatment of 5- to 17-year-old children with autism (NCT00086645, http://www.clinical trials.gov/ct/show/nct00086645?order=2).

\section{Measurement and Treatment Research to Improve Cognition in Schizophrenia}

The Measurement and Treatment Research to Improve Cognition in Schizophrenia (MATRICS) program is an example of a collaborative NIMH/industry/academic approach to advance drug development by focusing on a core component of the disorder, the neurocognitive impairments (http://www.matrics.ucla.edu/). The goals of this program were to catalyze regulatory acceptance of cognition in schizophrenia as a target for drug registration, promote development of novel compounds to enhance cognition in schizophrenia, leverage economic research power of industry to focus on important but neglected clinical targets, and identify lead compounds that support proof of concept trials for cognitive enhancement in schizophrenia. A series of conferences were held as part of the MATRICS process to assess what is known about cognitive deficits in schizophrenia, develop a consensus on the promising targets for intervention and the most promising models (animal and human) for use in drug development for this indication, identify a core battery of cognitive assessment tests, and identify the most appropriate clinical trials design. These conferences included participants from NIMH, the FDA, academia, and industry. The major outcome was the identification of seven key domains of cognitive function that are disrupted in schizophrenia and the development of a consensus test battery to assess those domains for use in clinical trials of pro-cognitive medications for schizophrenia (Buchanan et al, 2005). Key initiatives and outcome are summarized below. For a recent review of the program and targets for cognitive enhancement in schizophrenia, see Gray and Roth, 2007; Marder and Fenton, 2004; Stover et al, 2007.

MATRICS Consensus Cognitive Battery, Part 1: Test Selection, Reliability, and Validity. The lack of an accepted standard for measuring cognitive change in schizophrenia has been a major obstacle to regulatory approval of cognition-enhancing treatments. A primary mandate of the NIMH MATRICS initiative was to develop a consensus cognitive battery for clinical trials of cognition-enhancing treatments for schizophrenia through a broadly based scientific evaluation of measures. The article by Nuechterlein et al (2008) reports the process by which 10 tests were selected to represent seven cognitive domains in the MATRICS Consensus Cognitive Battery (MCCB); http:// www.matrics.ucla.edu/matrics-psychometrics-frame.htm. This is expected to be the standard tool for assessing cognitive change in clinical trials of cognition-enhancing drugs for schizophrenia. It may also aid evaluation of cognitive remediation strategies.

MATRICS Consensus Cognitive Battery, Part 2: CoNorming and Standardization. To facilitate interpretation of results from the MCCB using a common scaling across tests, normative data were obtained from a single representative US community sample of 300 individuals and the components were co-normed with allowance for 
demographic corrections, facilitating valid interpretation of test scores and communication of findings across studies (Kern et al, 2008). These normative data will aid in interpreting MCCB test results during clinical trials of cognition-enhancing agents.

\section{NIMH EFFORTS TO EXPAND TOOLS FOR ASSESSING COGNITIVE IMPAIRMENTS AND NEGATIVE SYMPTOMS IN SCHIZOPHRENIA}

Although the MATRICS initiative led to the development of a consensus cognitive battery to assess cognitive change in clinical trials, many of the neuropsychological measures included in the MATRICS battery are complex. There is a need for more precise assessment instruments to measure changes in cognitive functioning in treatment studies that incorporate advances in experimental cognitive science and cognitive neuroscience methods. The goal of the Adapting Basic Cognitive Measures for Clinical Assessment of Schizophrenia initiative is to support the selection, adaptation, and optimization of experimental cognitive measures for use in treatment trials of schizophrenia (http://grants. nih.gov/grants/guide/rfa-files/RFA-MH-08-090.html).

\section{Cognitive Neuroscience Approaches to Treatment Development for Impaired Cognition in Schizophrenia}

The Cognitive Neuroscience Approaches to Treatment Development for Impaired Cognition in Schizophrenia (CNTRICS) initiative supports a series of workshops as a continuation of MATRICS. The goal of the CNTRCS workshops is to develop, adapt, and implement measurement approaches from cognitive, social, and affective neuroscience for use in clinical trials (http://cntrics.ucdavis.edu/). The current clinical tests used to assess cognition in schizophrenic patients and the effects of therapeutics on cognitive function were developed before basic research in cognitive neuroscience identified specific neural circuits and systems potentially involved in cognitive processes. Cognitive neuroscientists have developed animal models and testing paradigms that tap into the basic neural mechanisms believed to underlie cognition, however, these paradigms have not, for the most part, been used in the drug development process and have not been translated to the clinical setting. The workshops are intended to help develop tests that are more specific measures of brain functions related to cognitive function in schizophrenia and to assure that these measures are validated for use in clinical trials. For example, a cognitive neuroscience approach is being used to examine the function of specific cognitive systems (Carter and Barch, 2007). These theoretical model systems have been conceptualized, specified in mechanistic terms and validated using exhaustive experimentation in which task parameters are varied and performance measured to test predictions arising from the models. Importantly, these constructs have been increasingly defined in terms of brain function, using noninvasive measures such as evoked response potentials and fMRI to characterize the functioning of well-specified cognitive systems in the human brain. Further, many of these constructs have also been validated in animal models that have allowed very precise specification of the pattern of neural activity, supporting distinct cognitive mechanisms in specific brain regions and the neuropharmacological substrates of these mechanisms.

\section{Negative Symptoms}

A consensus-building meeting sponsored by NIMH indicated that negative symptom in schizophrenia is another area where there is a need for treatment development. The group agreed that the development of new therapeutic approaches could be aided by a data-driven approach, similar to MATRICS, for developing a new instrument. A workgroup chaired by Dr Brian Kirkpatrick is evaluating the Negative Symptom Rating Scale to improve the measurement of negative symptoms (http://www.matrics. ucla.edu/matrics-neg-symptom-initiative.shtml). Training tapes for this instrument have been distributed to a number of investigators. These will be administered to schizophrenia populations. After some refinement, systematic data collection will be initiated to evaluate the psychometric characteristics of the instrument (Kirkpatrick et al, 2006; Stover et al, 2007).

\section{Treatment Units for Research on Neurocognition and Schizophrenia}

Treatment Units for Research on Neurocognition and Schizophrenia (TURNS) is another NIMH strategy to stimulate academic- and industry-sponsored research focused on cognitive deficits in schizophrenia (http:// www.turns.ucla.edu). The TURNS program supports a clinical research network to evaluate the safety, efficacy, pharmacokinetics, and pharmacodynamics of new agents for the treatment of cognitive deficits of schizophrenia using the MCCB. The TURNS program also provides support for a compound selection committee to review compounds with putative cognitive enhancing activity for proof of concept studies for the treatment of cognitive deficits of schizophrenia within the network (http:// www.turns.ucla.edu/turns-compound-frame.htm). To date, two compounds have been selected to move forward for testing in the TURNS clinical trials network.

The first is AL-108, an intranasal drug product containing NAP, an eight amino-acid peptide (Asn-Ala-Pro-Val-SerIle-Pro-Gln; NAPVSIPQ) fragment of the much larger activity-dependent neuroprotective protein known to participate in neurodevelopment and neuroprotection, mediating its effects in part through interaction with microtubules (http://www.allontherapeutics.com/pd_AL-108.htm). AL-108 is associated with microtubule interaction and neuroprotection and has been shown to cross the bloodbrain barrier. A 12-week multicenter, double-blind clinical trial of two doses of AL-108 vs placebo is underway, with 
AL-108 being added as an adjunct to schizophrenia patients' antipsychotic medication (NCT00505765, http://clinicaltrials. gov/ct2/show/NCT00505765?term=AL-108\&rank=1).

A proof of concept study will test the GABA-A $\alpha 2$ mechanism of cognitive enhancement as an adjunctive treatment for schizophrenia. Two doses of MK-0777 will be tested for efficacy and safety in the treatment of cognitive impairments in schizophrenia in a 6-week multicenter, doubleblind clinical trial. Secondary goals are to determine whether MK-0777 has beneficial effects on measures of functional capacity and patient self-report of cognitive function (NCT00505076, http://clinicaltrials.gov/ct2/show/NCT00505076? term $=$ MK-0777\&rank=1). It is anticipated that TURNS will conduct future trials of clinical candidates with promising cognitive enhancing activity that will be sponsored by industry.

\section{NIMH SUPPORT FOR REAL-WORLD OUTCOMES RESEARCH STUDIES}

Measurement tools used to assess cognitive performance of individuals in clinical trials are not able to predict improvement in the ability to function in real-life tasks. NIMH has undertaken several initiatives, in partnership with academia and industry, to identify and test real-world outcome measures that can be used in clinical trials of cognitive enhancing agents in schizophrenia.

\section{Functional Co-primary Measures for Clinical Trials in Schizophrenia}

During the consensus meeting of the NIMH MATRICS initiative, the FDA took the position that a cognitiveenhancing drug for schizophrenia should show changes on: (1) an accepted consensus cognitive performance measure and (2) an additional measure (ie, a co-primary) that is considered functionally meaningful. In the MATRICS Psychometric and Standardization Study, two measures of functional capacity and two interview-based measures of cognition were evaluated in 176 patients with schizophrenia. It was decided not to endorse a single co-primary measure of community function for clinical trials at this point. The current findings offer the initial steps to identify functionally meaningful co-primary measures in this area and will help to guide further evaluation of such measures (Bellack et al, 2007).

MATRICS Co-primary and Translation (MATRICS-CT) is another example of a public-private partnership involving NIMH, academia, and industry. MATRICS-CT will identify and evaluate potential 'co-primary' measures that can be used in clinical trials to assess functionally meaningful outcomes (ie, the ability of individuals to perform a functionally meaningful task and whether this improvement is related to changes in their community functioning). In addition, the project will translate the MCCB into foreign languages to facilitate international clinical trials. Unlike MATRICS, this project was initiated and is supported by industry and is coordinated by the Foundation for the NIH
(FNIH). For additional information, see http://www. esi-topics.com/fmf/2007/september07-MichaelFGreen.html).

Recently, experts in neurocognition, outcomes, and clinical trials from academia, government, and industry held a series of conferences and a live meeting to begin the process of selecting the measures that could be included in a validation study. Criteria for selecting measures were developed and tests were nominated by researchers and clinicians. Measures that are based on well-defined tasks carried out by patients (or performance-based measures) were evaluated as well as measures that depend on an interview. In February 2008, a diverse group of experts from academia and industry reviewed information about each measure and rated how they met the selection criteria. The results of the meeting are posted at http://www.matrics. ucla.edu. The information from the meeting is being used to design a validation study.

In a related effort, the RAND panel method is being used in an NIMH program that focuses on selecting measures of 'real-world' outcome in schizophrenia (CRISP abstract, http:// crisp.cit.nih.gov/crisp/CRISP_LIB.getdoc?textkey $=7258655$ \&p_grant_num $=1$ R01MH078775-01 A\&p_query=\&ticket $=$ 58950112\&p_audit_session_id=297411474\&p_keywords=). Using a similar process for developing criteria for measures, a panel was convened in February 2008. Again, agreement was reached on rating how nominated measures met selection criteria. The ratings are being reviewed and a validation study is being planned.

Two recently funded grants addressing this issue aim to identify existing measurement methods that most accurately assess schizophrenic participants' skills and their actual application of those skills and function in daily-life situations. Results of the project are meant to provide scientists who conduct future research on the effectiveness of treatments with tools that will reflect the truest possible picture of daily-life outcomes in schizophrenia (http:// www.nimh.nih.gov/science-news/2008/real-world-outcomesin-schizophrenia-are-focus-of-two-new-nimh-grants.shtml).

\section{COMPLIMENTARY NIH EFFORTS TO DEVELOP STANDARDIZED ASSESSMENT TOOLS}

The NIH Toolbox for Assessment of Neurological and Behavioral Function is a comprehensive set of behavioral measures for assessing cognition, emotion, sensation, and motor functions (http://neuroscienceblueprint.nih.gov/ neuroscience_resources/clinical_tools.htm; http://www. nihtoolbox.org/default.aspx). The goal of the Toolbox is to provide uniformity and enhance comparability across human clinical neuroscience studies, and it may be additionally advantageous for treatment trials. Further specification of such measures across species, and also across developmental stages, could be of significant use in preclinical drug development to advance the specificity of pharmacological treatments for children and adolescents. 
Patient-Reported Outcomes Measurement Information System (PROMIS) is another notable example of an NIHwide program designed to improve the measurement of health outcomes provided by patient report (www.nihpromis. org). The broad objectives of PROMIS are to: (1) develop and test a large bank of items measuring patient-reported outcomes; (2) create a computerized adaptive testing system that allows for efficient, psychometrically robust assessment of patient-reported outcomes in clinical research involving a wide range of chronic diseases; and (3) create a publicly available system that can be added to and modified periodically and that allows clinical researchers to access a common repository of items and computerized adaptive tests. Currently in year 4 of the 5-year project, the PROMIS network has developed item banks measuring physical functioning, pain, fatigue, depression, anxiety, anger, and social role functioning and satisfaction. These item banks were tested and calibrated in over 20000 participants from general and clinical populations, and these banks show superior precision compared to existing scales. The PROMIS Assessment Center was developed to provide computerized administration of these item banks by a fully automated patient reporting system. PROMIS has developed a number of collaborations with other federally funded projects, test developers, and early adopters. The PROMIS network is currently working on establishing a public-private partnership to sustain PROMIS services and expand the research efforts beyond the project period.

\section{FUTURE DIRECTIONS AND CLINICAL IMPLICATIONS}

\section{Optimizing the Preclinical/Clinical Interface}

Through individual and government efforts such as the Molecular Libraries, NCDDG, CDDG, MATRICS, and TURNS, unprecedented opportunities exist to identify novel targets (molecular and cellular as well as core domains of dysfunction) and novel compounds as potential starting points for the discovery of new mechanism of action treatments for psychiatric disorders. This opportunity also presents unique challenges, including more rigorous target validation (from target identification to clinical validation), better models to predict both efficacy and adverse effects in humans, new diagnostic approaches that may account for greater patient heterogeneity in treatment response, and refined tools and biomarkers for identifying specific defects in brain associated with disorders at the molecular, cellular, circuit, and ultimately the behavioral levels. Efforts exemplified by MATRICS, CNTRICS, and PROMIS are essential for identifying key behavioral and physiological measures of illness and treatment response that can be implemented in real-world populations. The development of biomarkers, though still in its infancy, will have enormous impact on the successful integration of these approaches toward the development of treatments for psychiatric disorders.

\section{Development of Biomarkers for Mental Disorders}

As previously discussed, the greatest impediment in the development of reliable model systems for drug discovery relevant to psychiatric disorders is the inherent discontinuity between models and imprecise diagnostic methods or indicators of the pathophysiology of psychiatric disorders. The identification, validation, and utilization of biomarkers that serve as quantifiable indicators of normal biological processes, pathophysiological states, and responses to therapeutics will be indispensable tools for generating reliable proof of mechanism and proof of concept data in preclinical and clinical studies. The ability to reliably identify individuals with specific deficits is expected to be a major advance for both clinical research and treatment development. Biomarkers will be identified from efforts to develop well-defined psychometric, neuroimaging, genetics, and proteomics measures, from modeling and simulating preclinical and early clinical findings, and other approaches supported through NIMH and NIH initiatives and programs.

The Biomarkers Consortium is a public-private biomedical research partnership managed by the FNIH. The Biomarkers Consortium embraces government, industry, patient advocacy groups, and other nonprofit private sector organizations to search for and validate new biomarkers to accelerate the competitive delivery of successful new technologies, medicines, and therapies for prevention, early detection, diagnosis, and treatment of disease (http:// www.biomarkersconsortium.org/). In the area of psychiatry, the Biomarkers Consortium currently supports a WGAS in major depressive disorder to identify genomic biomarkers for treatment response, as an extension of the NIMH supported Sequenced Treatment Alternatives to Relieve Depression (STAR $\left.{ }^{\star} \mathrm{D}\right)$ study (http://www.fnih.org/news/ news_events_Oct.shtml; http://www.nimh.nih.gov/health/ trials/practical/stard/index.shtml), and there are other CNS projects under consideration. The Biomarkers Consortium invites project submissions in all therapeutic areas (BC proposals, http://www.biomarkersconsortium.org/index. php? option=com_content\&task=section\&id=7\&Itemid=41). This ambitious public-private partnership is anticipated to have substantial biomedical impact by its efforts to identify and qualify biomarkers to support basic and translational research, guide clinical practice and, ultimately, support the development of safe and effective medicines and treatments.

\section{Modeling Genetic, Developmental, and Environmental Risk Factors}

Additional large efforts to identify genes associated with mental disorders are being supported by $\mathrm{NIH}$ and other agencies. For example, through the Autism Consortium, NIH is supporting studies aimed at identifying genes associated with autism spectrum disorders (http://www.nimh.nih.gov/ press/autismconsortiumgrants.cfm). NIH supports WGAS to identify genetic factors influencing risk for complex diseases to facilitate discovery of new molecular targets for prevention, diagnosis, and treatment through the Genetic 
Association Information Network (GAIN, http://www.fnih. org/GAIN2/home_new.shtml). The first phase of GAIN is genotyping samples from studies in schizophrenia, bipolar disorder, ADHD, and major depression (http://www.fnih. org/GAIN2/initial_studies.shtml). The genotype and associated de-identified phenotype information will be deposited in dbGAP, a database of genotype and phenotype information managed by the National Library of Medicine (http://www.ncbi.nlm.nih.gov/sites/entrez?Db = gap) for access by the research community (http://grants.nih.gov/ grants/guide/notice-files/NOT-OD-07-066.html). NIH also supports genotyping and other genomic research methodologies to identify the major susceptibility and etiologic factors for complex diseases of significant public health impact through the Genes, Environment and Health Initiative (GEI, http://www.genome.gov/19518663).

These NIH efforts are complimentary to private efforts. For example, the Stanley Center for Psychiatric Research was recently founded at the Broad Institute of MIT and Harvard University by a grant from the Stanley Medical Foundation. The broad-based goals of this program are to identify and characterize risk genes for psychiatric diseases through linkage and association studies in the human population, develop animal and cellular models to investigate the function of candidate genes and pathways, develop sophisticated imaging techniques for elucidating brainbased phenotypes in clinical disease, and to design highthroughput chemical screens for identifying molecules that modulate important cellular targets related to neural function (http://www.broad.mit.edu/psych/).

\section{Application of Genetic Data for Target Identification and Validation for Drug Discovery}

It is still too early to assess the impact of the anticipated explosion of genetic data from GAIN, GEI, WGAS, and other genetic studies on the field of psychiatric drug discovery. As genetic and clinical evidence accumulates for risk genes for psychiatric disorders, it will be important to understand the biology and function of gene variants and gene-dosage abnormalities, and to develop a strategy for prioritizing research investment in the large number of emerging variants. As an example of the potential impact of WGAS on T1 research, disrupted-in-schizophrenia 1 (DISC1) has been identified as one of the most compelling risk genes for schizophrenia and other major mental disorders. Research to determine the expression pattern of DISC1 in brain, and RNA interference studies to assess its function has enabled an understanding of the involvement of DISC1 in neuronal development and cell signaling. Innovative technology and informatics approaches have identified DISC1-binding partners, including Ndel, phosphodiesterase $4 \mathrm{~B}$, and other molecules found within a large DISC1 interaction network, the 'DISC1 interactome' (Wang Q et al, 2008). Research on the biology of DISC1 has lead to the identification of novel targets for drug discovery, the development of transgenic mouse models expressing DISC1 variants, and interest in the development of mice expressing DISC1 interactors for testing compounds as potential clinical candidates for schizophrenia.

Another example is the identification of the metabotropic glutamate receptor 5 subtype (mGluR5) as a target for drug discovery and development for treatment of fragile $\mathrm{X}$ syndrome, the most common inherited form of mental retardation. Recent studies in mice lacking the FMRX protein provide compelling data that reducing mGluR5 receptor levels can reverse both brain and behavioral abnormalities (Dolen and Bear, 2008). NIMH is providing support to Seaside Therapeutics, along with co-funding from two additional neuroscience institutes, to test the toxicology and safety of a novel mGluR5 antagonist, STX107, in animals (http://www.nimh.nih.gov/sciencenews/2007/study-aims-to-develop-first-medications-for-fragile$\mathrm{x}$-syndrome-leading-inherited-cause-of-mental-retardation. shtml). This is yet another example of NIH funding to bridge the 'valley of death' for testing a novel compound for an orphan disease. If additional tests in this study confirm the compound's safety, the scientists will request FDA approval to conduct research that will determine dosage and safety in normal, human volunteers. Success in phase I studies will allow the compound to secure venture capital/venture philanthropic funding to support clinical trials of the novel mGluR5 mechanism to treat fragile $\mathrm{X}$ syndrome.

\section{Challenges}

The intent of this chapter is to provide perspective on NIMH efforts to facilitate the discovery and use of novel therapeutic approaches for mental disorders. The MATRICS and CNTRICS initiatives represent a new approach to facilitate the development of treatments that are targeted toward improving core domains of dysfunction and real-world functioning of individuals with mental disorders. Although progress has been made, regulatory acceptance of cognitive enhancement as a target for drug registration in schizophrenia awaits validation of co-primary measures that reflect changes in real-world function. Similar approaches are needed for negative symptoms in schizophrenia and for bipolar disorder where the search for therapies that target core domains of dysfunction may be even more challenging. Existing preclinical models are variable in their degree of predictive value in assessing potential efficacy or adverse effects of new compounds in psychiatric disorders, calling for a new approach to conceptualize models and their place in the drug discovery pipeline. Continued as well as new efforts to support research aimed at understanding the genetic, molecular, and physiological basis of specific mental disorders are likely to illuminate new paths and approaches for developing and validating models to assess drug efficacy in animals and humans.

\section{SUMMARY}

In closing, this article has highlighted $\mathrm{NIMH}$ and $\mathrm{NIH}$ programs and public-private partnerships that boldly extend 
research support into the area of drug discovery and proof of concept trials, a critical first step in developing new treatments for patients with psychiatric disorders that are effective at both the bedside (T1) and in clinical practice (T2). Continued emphasis on such early phase initiatives should accelerate the transfer of biomedical discoveries into a wider range of testable interventions for different brain targets, including some that may correspond to symptom domains that cut across conventional diagnostic categories. Although the focus was on programs, initiatives, and emerging opportunities for translational research in psychiatric drug discovery and development, there are numerous NIH and institute programs that provide support for discovery and development in other CNS therapeutic areas. In keeping with the grand challenge, 'Now is the time for research to set an ambitious goal of finding cures and preventive interventions for these disabling illnesses' (Insel and Scolnick, 2006), the transition of clinical candidates from discovery to clinical development can be accelerated if a new paradigm is adopted to bring the collective intellectual resources of public and private investigators to bear at every stage of the discovery and development pipeline.

Although the current status of therapeutic discovery for psychiatric disorders has clearly not progressed rapidly enough, significant advancements in genetics, molecular neuroscience, and systems neurobiology are poised to present novel treatment targets and lead molecules. At the same time, improved identification of critical behavioral and physiological parameters of illness and treatment response have potential to greatly improve clinical determination of efficacy and to guide the development of more predictive model organisms or systems. Full implementation of these approaches will require greater communication among research communities and with more traditional drug development enterprises. To forge paths where discovery in basic and clinical research ultimately intersect with and impact the treatment of psychiatric disorders, greater translational communication is also needed between the research community and providers of mental heath services. The next article in this series (Wang PS et al, 2008) describes some of the unique challenges in the $\mathrm{T} 2$ studies and how to improve the trajectories of advances in both $\mathrm{T} 1$ and $\mathrm{T} 2$ to improve treatment.

\section{DISCLOSURE/CONFLICT OF INTEREST}

The authors declare that, except for income received from the NIMH, no financial support or compensation has been received from any individual or corporate entity over the past 3 years for research or professional service and there are no personal financial holdings that could be perceived as constituting a potential conflict of interest.

\section{REFERENCES}

Armburster BN, Li X, Pausch MH, Herlitze S, Roth BL (2007). Evolving the lock to fit the key to create a family of $\mathrm{G}$ protein-coupled receptors potently activated by an inert ligand. Proc Natl Acad Sci USA 104: 5163-5168.
Agid Y, Buzsaki G, Diamond DM, Frackowiak R, Giedd J, Girault JA et al (2007) How can drug discovery for psychiatric disorders be improved? Nat Rev Drug Discov 6: 189-201.

Austin CP, Brady LS, Insel TR, Collins FS (2004). NIH molecular libraries initiative. Science 306: 1138-1139.

Bellack AS, Green MF, Cook JA, Fenton W, Harvey PD, Heaton RK et al (2007). Assessment of community functioning in people with schizophrenia and other severe mental illnesses: a white paper based on an $\mathrm{NIMH}$-sponsored workshop. Schizophr Bull 33: 805-822.

Brady LS, Giffin RB, Woodcock J, Cassell GH, Holmes EW (2008). Grand challenges for psychiatric drug discovery: a perspective. Neuropsychopharmacology 33: 2047.

Buchanan RW, Davis M, Goff D, Green MF, Keefe RS, Leon AC et al (2005). A summary of the FDA-NIMH-MATRICS workshop on clinical trial design for neurocognitive drugs for schizophrenia. Schizophr Bull 31: 5-19.

Carpenter WT, Koenig Jl (2008). The evolution of drug development in schizophrenia: past issues and future opportunities. Neuropsychopharmacology 33: 2061-2079.

Carter CS, Barch DM (2007). Cognitive neuroscience-based approaches to measuring and improving treatment effects on cognition in schizophrenia: the CNTRICS initiative. Schizophr Bull 33: 1131-1137.

Chong CR, Sullivan Jr DJ (2007). New uses for old drugs. Nature 448 645-646.

Conn PJ, Roth BL (2008). Opportunities and challenges of psychiatric drug discovery: roles for scientists in academic, industry, and government settings. Neuropsychopharmacology 33: 2048-2060.

Dolen G, Bear MF (2008). Role for metabotropic glutamate receptor 5 (mGluR5) in the pathogenesis of fragile X syndrome. J Physiol 586: 1503-1508.

Felger JC, Alagbe O, Hu F, Mook D, Freeman AA, Sanchez MM et al (2007). Effects of interferon-alpha on rhesus monkeys: a nonhuman primate model of cytokineinduced depression. Biol Psychiatry 62: 1324-1333.

Gray JA, Roth BL (2007). Molecular targets for treating cognitive dysfunction in schizophrenia. Schizophr Bull 33: 1100-1119.

Huang W, Zheng W, Urban DJ, Inglese J, Sidransky E, Austin CP et al (2007). N4phenyl modifications of N2-(2-hydroxyl)ethyl-6-(pyrrolidin-1-yl)-1,3,5-triazine-2,4diamines enhance glucocerebrosidase inhibition by small molecules with potential as chemical chaperones for Gaucher disease. Bioorg Med Chem Lett 17: 5783-5789.

Hughes B (2008). 2007 FDA drug approvals: a year of flux. Nat Rev Drug Discov 7 : 107-109.

Huryn DM, Cosford NDP (2008). The molecular libraries screening center network: identifying chemical probes of biological systems. Annu Rep Prog Chem, Sect A: Inorg Chem 42: 401-416.

Insel TR (2007). From animal models to model animals. Biol Psychiatry 62 1337-1339.

Insel TR, Scolnick EM (2006). Cure therapeutics and strategic prevention: raising the bar for mental health research. Mol Psychiatry 11: 11-17.

Kern RS, Nuechterlein KH, Green MF, Baade LE, Fenton WS, Gold JM et al (2008). The MATRICS consensus cognitive battery, part 2: co-norming and standardization. Am J Psychiatry 165: 214-220.

Kirkpatrick B, Fenton WS, Carpenter Jr WT, Marder SR (2006). The NIMH-MATRICS consensus statement on negative symptoms. Schizophr Bull 32: 214-219.

Lazo JS, Brady LS, Dingledine R (2007). Building a pharmacological lexicon: smal molecule discovery in academia. Mol Pharmacol 72: 1-7.

Lewis LM, Sheffler D, Williams R, Bridges TM, Kennedy JP, Brogan JT et al (2008). Synthesis and SAR of selective muscarinic acetylcholine receptor subtype 1 (M1 mAChR) antagonists. Bioorg Med Chem Lett 18: 885-890.

Lopez AD, Mathers CD, Ezzati M, Jamison DT, Murray CJL (2006). Global Burden of Disease and Risk Factors. Oxford University Press/World Bank: New York.

Mailman RB (2007). GPCR functional selectivity has therapeutic impact. Trends Pharmacol Sci 28: 390-396.

Malberg JE, Platt B, Rizzo SJ, Ring RH, Lucki I, Schechter LE et al (2007). Increasing the levels of insulin-like growth factor-I by an IGF binding protein inhibitor produces anxiolytic and antidepressant-like effects. Neuropsychopharmacology 32: 2360-2368.

Marder SR, Fenton W (2004). Measurement and treatment research to improve cognition in schizophrenia: NIMH MATRICS initiative to support the development of agents for improving cognition in schizophrenia. Schizophr Res 72: 5-9.

Mathew SJ, Manji HK, Charney DS (2008). Novel drugs and therapeutic targets for severe mood disorders. Neuropsychopharmacology 33: 2080-2092.

Moran N (2007). Public sector seeks to bridge 'valley of death'. Nat Biotechnol 25: 266. Niswender CM, Johnson KA, Luo Q, Ayala JE, Kim C, Conn PJ et al (2008). A novel assay of $\mathrm{Gi} / \mathrm{o}$-linked $\mathrm{G}$ protein coupled receptor coupling to potassium channels provides new insights into the pharmacology of the group III metabotropic glutamate receptors. Mol Pharmacol 73: 1213-1224. 
Nuechterlein KH, Green MF, Kern RS, Baade LE, Barch DM, Cohen JD et al (2008). The MATRICS consensus cognitive battery, part 1: test selection, reliability, and validity. Am J Psychiatry 165: 203-213.

Pangalos MN, Schechter LE, Hurko O (2007). Drug development for CNS disorders: strategies for balancing risk and reducing attrition. Nat Rev Drug Discov 6: 521-532.

Skoumbourdis AP, Huang R, Southall N, Leister W, Guo V, Cho MH et al (2008). Identification of a potent new chemotype for the selective inhibition of PDE4. Bioorg Med Chem Lett 18: 1297-1303.

Stover EL, Brady L, Marder SR (2007). New paradigms for treatment development. Schizophr Bull 33: 1093-1099.

Sung NS, Crowley Jr WF, Genel M, Salber P, Sandy L, Sherwood LM et al (2003). Central challenges facing the national clinical research enterprise. JAMA 289: 1278-1287.
Titus S, Neumann S, Zheng W, Southall N, Michael S, Klumpp C et al (2008). Quantitative high-throughput screening using a live-cell cAMP assay identifies small-molecule agonists of the TSH receptor. J Biomol Screen 13: 120-127.

Urban JD, Clarke WP, von Zastrow M, Nichols DE, Kobilka B, Weinstein $\mathrm{H}$ et al (2007). Functional selectivity and classical concepts of quantitative pharmacology. J Pharmacol Exp Ther 320: 1-13.

Vitiello B (2007). Research in child and adolescent psychopharmacology: recent accomplishments and new challenges. Psychopharmacology (Berl) 191: 5-13.

Wang PS, Heinssen R, Oliveri ME, Wagner A, Goodman W (2008). Bridging bench and practice: translational research for schizophrenia and other psychotic disorders. Neuropsychopharmacology Reviews (in press).

Wang Q, Jaaro-Peled H, Sawa A, Brandon NJ (2008). How has DISC1 enabled drug discovery? Mol Cell Neurosci 37: 187-195. 